\title{
Alocação preferencial de recursos e morfologia de órgãos subterrâneos em plantas resistentes ao fogo em vegetação campestre
}

\author{
Miriã Maria Almeida de Abreu Silva Ferreira* \& Abel Augusto Conceição
}

Laboratório Flora e Vegetação, Departamento de Ciências Biológicas, Universidade Estadual de Feira de Santana, Av. Transnordestina, s.n., Novo Horizonte, 44036-900, Feira de Santana, Bahia, Brasil.

\begin{abstract}
Resumo - Os sistemas subterrâneos são importantes para sobrevivência das plantas e suas características morfológicas têm significado especial em ambientes sob distúrbio. O presente estudo teve como objetivo avaliar a existência de alocação preferencial dos recursos nas partes subterrâneas de plantas resistentes ao fogo e discutir implicações da alocação de recursos e da morfologia na dinâmica espacial e temporal de comunidades campestres suscetíveis ao fogo. $\mathrm{O}$ estudo restringiu-se a seis espécies campestres, sendo três monocotiledôneas (Hypolytrum pulchrum, Echinolaena inflexa e Andropogon sp.) e três eudicotiledôneas (Euphorbia sarcodes, Eryngium paraguariense e Marcetia taxifolia). As caracterizações morfológicas foram realizadas em cerca de cinco indivíduos de cada espécie, a partir de cortes anatômicos, fotos, medições com paquímetro digital, trenas e quantificação de biomassa seca das partes aéreas e subterrâneas. As plantas apresentaram diferentes arranjos espaciais que permitem a coexistência no espaço através de um jogo de habilidades associadas a características morfofuncionais aéreas e subterrâneas integradas. A maioria das espécies apresentou sistema subterrâneo integrado em redes com alocação preferencial de recursos para partes aéreas, com exceção de E. sarcodes. A morfologia é um fator fundamental nas respostas competitivas. As gemas desenvolvidas principalmente nos rizomas de plantas abundantes nessas fisionomias abertas constituem uma evidência da grande importância dessas estruturas na dinâmica de comunidades campestres.

Palavras-chave adicionais: biomassa, Chapada Diamantina, respostas competitivas, sistemas subterrâneos, sobrevivência.

Abstract (Preferential allocation of resources and morphology of underground organs of plants resistant to fire in grassland vegetation) - The underground systems are important for survival of plants and their morphological characteristics have special meaning in disturbed environments. The present study evaluated the existence of preferential allocation of resources in the underground parts of plants resistant to fire and discuss implications of resource allocation and morphology in spatial and temporal dynamics of grassland communities susceptible to fire. The study was restricted to six species of grassland, three monocots (Hypolytrum pulchrum, Echinolaena inflexa and Andropogon sp.) and three eudicots (Euphorbia sarcodes, Eryngium paraguariense and Marcetia taxifolia). The morphological characterizations were performed in about five individuals of each species from anatomical cuts, photos, measurements with digital calipers, tape measures and quantification of biomass share of overhead and underground. The plants showed different spatial arrangements that allow the coexistence in space through a set of skills associated with morphofunctional features integrated aerial and underground parts. Most species showed underground system integrated into networks with preferential allocation of resources to aerial parts, except for E. sarcodes. The morphology is a crucial factor in the competitive responses. The buds developed mainly on rhizomes of plants abundant in these open areas are evidence of the importance of these structures in the dynamics of grassland communities.
\end{abstract}

Additional key words: biomass, Chapada Diamantina, competitive responses, survival, underground systems.

Os campos rupestres exibem grande heterogeneidade ambiental e uma vegetação peculiar associada a áreas rochosas e em solos arenosos rasos. Os sistemas subterrâneos encontram-se principalmente associados à diversidade de formas de vida, conforme a variação do substrato (Conceição \& Giulietti 2002; Kolbek \& Alves 2008), com grande importância das gemas subterrâneas no processo de regeneração da vegetação afetada por incêndios (Menezes et al. 1979; Neves \& Conceição 2010) e que de alguma forma influenciam nos processos de sucessão e competição (Costa 2010).

A teoria de competição de recurso prediz a dinâmica de interações de espécies baseada em dinâmicas de recurso e em características de uso de recurso de cada espécie (Tilman 1982). A análise da alocação da biomassa pode esclarecer

*Autora para correspondência: miriabio@yahoo.com.br

Editor responsável: Alessandro Rapini

Submetido em: 18 out. 2011; publicação eletrônica: 11 jun. 2012 questões a respeito do estabelecimento, produtividade e persistência de espécies perenes (Scheffer-Basso et al. 2002) sujeitas ao fogo. No entanto, as respostas morfológicas e fisiológicas das plantas podem ser mais relevantes que a observação somente da biomassa, para compreensão das habilidades competitivas das plantas em solos mais ou menos distróficos (Casper \& Jackson 1997; Cahill 2003; Grams \& Andersen 2007).

Nos campos rupestres, as espécies exibem baixa variação fenotípica quanto à alocação dos recursos, pois estão adaptadas a ambientes nutricionalmente pobres (Negreiros et al. 2009). O conhecimento não só sobre biomassa, mas especialmente sobre características inerentes a respostas reprodutivas e vegetativas, sobretudo das competitivas e de grupos funcionais, é necessário para se entender os processos e consequências de diferentes respostas (Cianciaruso et al. 2010). Padrões de alocação em espécies de campos rupestres são cruciais para restauração de áreas degradadas, uma vez que irão recuperar 
informações sobre adaptações ecofisiológicas para o ambiente hostil (Negreiros et al. 2009).

Como o padrão de ramificações das raízes é determinante para a eficiência na obtenção de água e nutrientes do solo e possui papel central na funcionalidade dos sistemas radiculares (Fitter \& Stickland 1991), a hipótese do presente estudo foi de que as espécies dominantes em vegetação campestre são resistentes ao fogo e alocam maior parte dos recursos para os sistemas subterrâneos integrados, sendo as variações morfológicas dos sistemas subterrâneos fundamentais nas respostas competitivas. Nesse sentido, o presente estudo avaliou a morfologia e a alocação de recursos nas partes subterrâneas de plantas campestres resistentes ao fogo, visando compreender suas implicações na dinâmica espacial e temporal de comunidades suscetíveis ao fogo.

\section{Material e Métodos}

Área de estudo. O estudo foi desenvolvido em uma área com fisionomia campestre de transição entre campo rupestre e campo limpo, situada no Orquidário do Pai Inácio (12 $27^{\prime}$ S, $41^{\circ} 28^{\prime} \mathrm{W}, 950$ m s.n.m), Área de Proteção Ambiental Marimbús-Iraquara, próximo ao Parque Nacional da Chapada Diamantin, município de Palmeiras, Bahia, Brasil. O clima na região é tropical, com estações secas e úmidas (Nimer 1989). O solo é ácido com textura variando de areia a areia franca, com baixos teores de nutrientes, e apresenta diferenças de profundidade, coloração e presença de muitas rochas afloradas, caracterizando um mosaico (Conceição 2006; Grillo 2008).

Material de estudo. A partir de um levantamento fitossociológico realizado na área de estudo (Costa 2010), foram selecionadas seis espécies abundantes (materialtestemunho depositados no Herbário da Universidade Estadual de Feira de Santana - HUEFS), sendo três monocotiledôneas: Hypolytrum pulchrum (Rudge) H.Pfeiff (Cyperaceae; G. Costa 4); Echinolaena inflexa (Poir.) Chase (Poaceae; G. Costa 177); e Andropogon sp. (Poaceae; G. Costa 230); e três eudicotiledôneas: Euphorbia sarcodes Boiss. (Euphorbiaceae; G. Costa 204); Eryngium paraguariense Urb. (Apiaceae; G. Costa 121); e Marcetia taxifolia (A.St-Hil.) DC. (Melastomataceae; G. Costa 217). No estudo de Costa (2010), houve o restabelecimento de $97 \%$ da cobertura aérea da comunidade de plantas depois de um ano da queimada experimental, sendo que os índices de valor de cobertura das seis espécies aqui estudadas, contabilizados antes e depois de um ano do fogo foram, respectivamente: 33,77 e 28,09 (E. inflexa); 12,62 e 15,34 (E. sarcodes); 10,13 e 9,98 (H. pulchrum); 9,24 e 4,41 (Andropogon sp.); 7,20 e 8,53 (E. paraguariense); 5,77 e 3,35 (M. taxifolia)(Costa 2010).

Coleta do material. Foram realizadas duas formas de escavações: a simples, caracterizada pela remoção completa da planta; e a progressiva, realizada em duas plantas de propagação clonal (H. pulchrum e M. taxifolia), caracterizada pela escavação iniciada a partir do broto mais novo até o mais antigo (Silveira 2001), visando observar a disposição modular do sistema sem retirá-lo por completo do solo. As coletas foram realizadas no início da estação seca (10 ago. 2008). As caracterizações foram feitas em cerca de cinco indivíduos de cada espécie, a partir de medições com paquímetro digital de $0,1 \mathrm{~mm}$ de resolução, trenas, ilustrações, registros fotográficos e cortes anatômicos para confirmação das estruturas caulinares e radiculares. As plantas foram acondicionadas em sacos de papel e posteriormente secas em estufa a $60^{\circ} \mathrm{C}$ por $72 \mathrm{~h}$, até atingirem peso seco constante. Em uma área queimada experimentalmente sob supervisão de brigadistas do ICMBio/IBAMA local, indivíduos das seis espécies foram marcados para observação do potencial de rebrota dos sistemas depois do fogo, analisado pela recuperação da parte aérea, sendo três de E. paraguariense e E. sarcodes, com um das demais espécies.

Biomassa. A “estratégia” de investimento na ocupação espacial foi definida a partir da quantificação da biomassa seca aérea e subterrânea, separadas e pesadas. Foram calculados os índices $\alpha^{1}$ e $\alpha^{2}$ mediante o quociente entre a massa seca aérea (MSA) pela massa seca subterrânea (MSS). Devido às dificuldades para delimitação espacial dos sistemas subterrâneos, presença de diferentes estádios de desenvolvimento dos módulos, além da perda significativa das raízes que se quebraram durante a remoção das plantas, os cálculos foram realizados apenas para $E$. paraguariense e E. sarcodes; as biomassas aéreas e subterrâneas das demais espécies foram determinadas apenas para uma unidade modular.

\section{Resultados e Discussão}

Indivíduos de todas as espécies marcadas rebrotaram conforme observação realizada sete meses após a passagem do fogo (Figura 1), exceto M. taxifolia, a única espécie com estolão (Figura 2). O padrão estrutural dominante foi o de rizomas variados (H. pulchrum, E. paraguariense, Andropogon sp. e E. inflexa; Figuras 3-5). Euphorbia sarcodes foi a única com raiz pivotante (Figura 6), o que lhe confere características particulares quanto ao mecanismo de sobrevivência.

Diferenças no comprimento e número de entrenós, assim como na orientação e ramificação das unidades que compõem os sistemas subterrâneos caulinares, determinam as morfologias cespitosa, estolonífera ou rizomatosa (Briske 1986; Klink 1994), sendo o fogo capaz de promover simplificações na arquitetura (Klink \& Silva 2001). A arquitetura dos sistemas subterrâneos desempenha papel importante nas respostas competitivas e ao fogo (Klink \& Silva 2001; Kolbek \& Alves 2008; Appezzato-da-Glória \& Cury 2011). Nos campos rupestres, foram encontrados sistemas contínuos em $H$. pulchrum, E. paraguariense, $M$. taxifolia, Andropogon sp. e E. inflexa, com possibilidade de resposta integradora e divisora. Na resposta divisora, todos os módulos são autônomos para sobrevivência, ou seja, a reprodução de cada módulo só depende das condições locais de recursos. Na resposta integradora, a dinâmica de recursos é partilhada entre módulos (Oborny et al. 2000). 

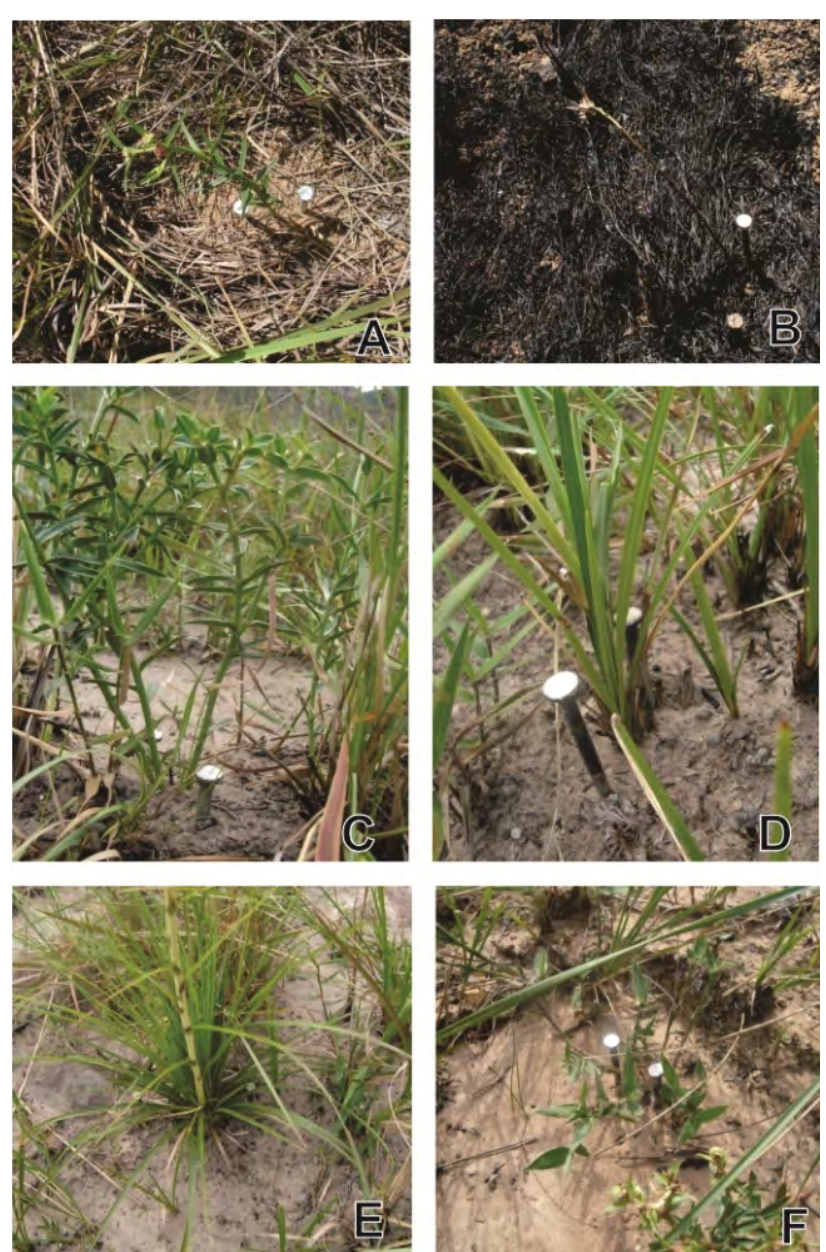

Figura 1. Plantas estudadas no Orquidário do Pai Inácio, município de Palmeiras, Chapada Diamantina, Bahia, Brasil. A-B. Euphorbia sarcodes: Antes (A) e imediatamente depois da queimada (B). C-F. Plantas que rebrotaram, sete meses depois do fogo: C- E. sarcodes; DHypolytrum pulchrum; E- Eryngium paraguariense; F- Echinolaena inflexa.

Sendo assim, o número e o comprimento dos entrenós podem influenciar na velocidade de resposta dos módulos ao fogo sob as variações de recursos no solo e a dinâmica conjunta do sistema pode garantir uma rebrota rápida pósfogo e ocupação estável do espaço em ambientes heterogêneos.

Segundo Negreiros et al. (2009), a variação e a composição do substrato nos campos rupestres têm uma forte influência sobre as variações fenotípicas quanto à alocação de recursos na planta. A biomassa do sistema subterrâneo de cada componente nem sempre é maior que a da parte aérea (Souza et al. 2010), como no caso da $E$. inflexa, que apresentou biomassa aérea de uma unidade modular 0,411 g e biomassa subterrânea 0,078 g. Entretanto, isso pode estar sendo compensado pela integração, como também ocorre em Androgogon sp., que apresenta uma morfologia cespitosa. Logo, nos campos rupestres, a mobilidade de recursos entre módulos em sistemas contínuos pode ser um fator importante para que as espécies clonais respondam à variação da distribuição espacial e temporal das condições e dos recursos, incluindo variações bióticas e distúrbios. Isso explicaria o fato das espécies marcadas terem rebrotado em tempos diferentes, como no caso da $M$. taxifolia, que teve rebrota tardia em relação às demais, com índice de valor de cobertura antes do fogo e um ano depois, respectivamente 5,77 e 3,35 (Costa 2010). Além disso, $M$. taxifolia foi a única que não apresentou rebrota local para um dos módulos marcados, sendo portanto, um indício de que houve uma dinâmica de recursos diferenciada, seja por fatores relacionados a condições locais de solo ou por direcionamento de recursos para módulos próximos. Isso foi possível de ser observado pela escavação progressiva.

Hypolytrum pulchrum. Apresenta um sistema de caules plagiotrópicos, disposto no solo em um complexo de redes em arcos de tamanhos variados, dos quais originam novos módulos, que podem ou não permanecer unidos por tempo longo (Figura 3). Os arcos se espalham rapidamente, são finos, tendo em média $2 \mathrm{~mm}$ de diâmetro quando jovens, e podem alcançar mais de $28 \mathrm{~cm}$ de profundidade; apresentam nós e entrenós marcantes e protegidos por catafilos. As raízes ficam principalmente na base de cada módulo na região nodal. A presença de arcos longos e curtos em $H$. pulchrum sugere duas respostas, forrageadora e "falangeal". A resposta forrageadora, por meio de arcos longos, tem efeito direto sobre a comunidade vegetal pela exploração de novas manchas de recursos (Oborny et al. 2000), aumentando a chance de competição interespecífica e diminuindo a intraespecífica, podendo ainda agir na exclusão competitiva de outras plantas (Bell \& Tomlinson 1980). Na forma de crescimento "falangeal", através de arcos curtos, a planta espalha-se lentamente na vegetação e permanece na área cerceando a entrada de outras plantas (van Groenendael et al. 1996). Nessas áreas, a competição intraespecífica deve ser maximizada e os arcos centrais expandem-se ilimitadamente.

O sistema de redes formado pela sobreposição dos arcos gera uma matriz firme no solo, conferindo função mecânica, evitando erosão e favorecendo a aeração do solo, podendo ainda impedir o crescimento de outras espécies onde exista alta concentração de $H$. pulchrum. A flexibilidade e curvatura dos arcos jovens podem ser úteis à aquisição de água e à facilidade de retorno à superfície.

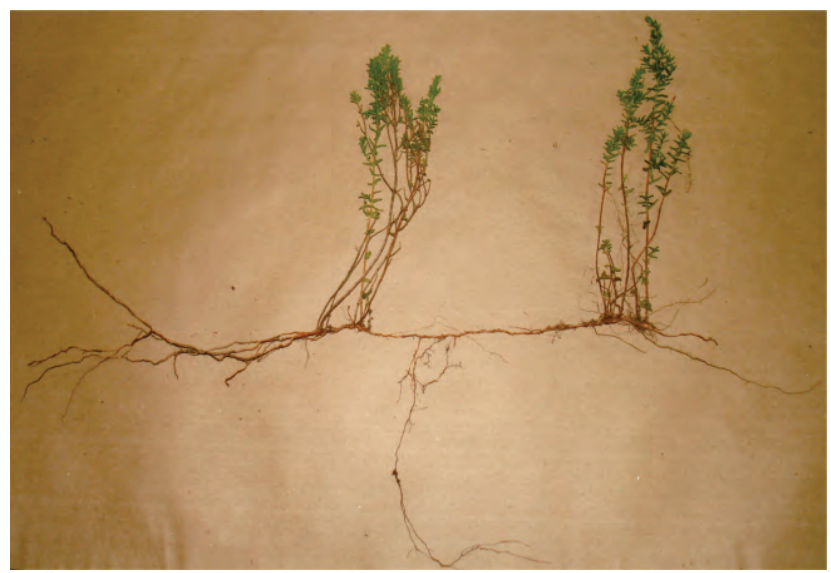

Figura 2. Estolão longo e esguio, com raízes pouco uniformes, de amostra de Marcetia taxifolia coletada no Orquidário do Pai Inácio, município de Palmeiras, Bahia, Brasil. 

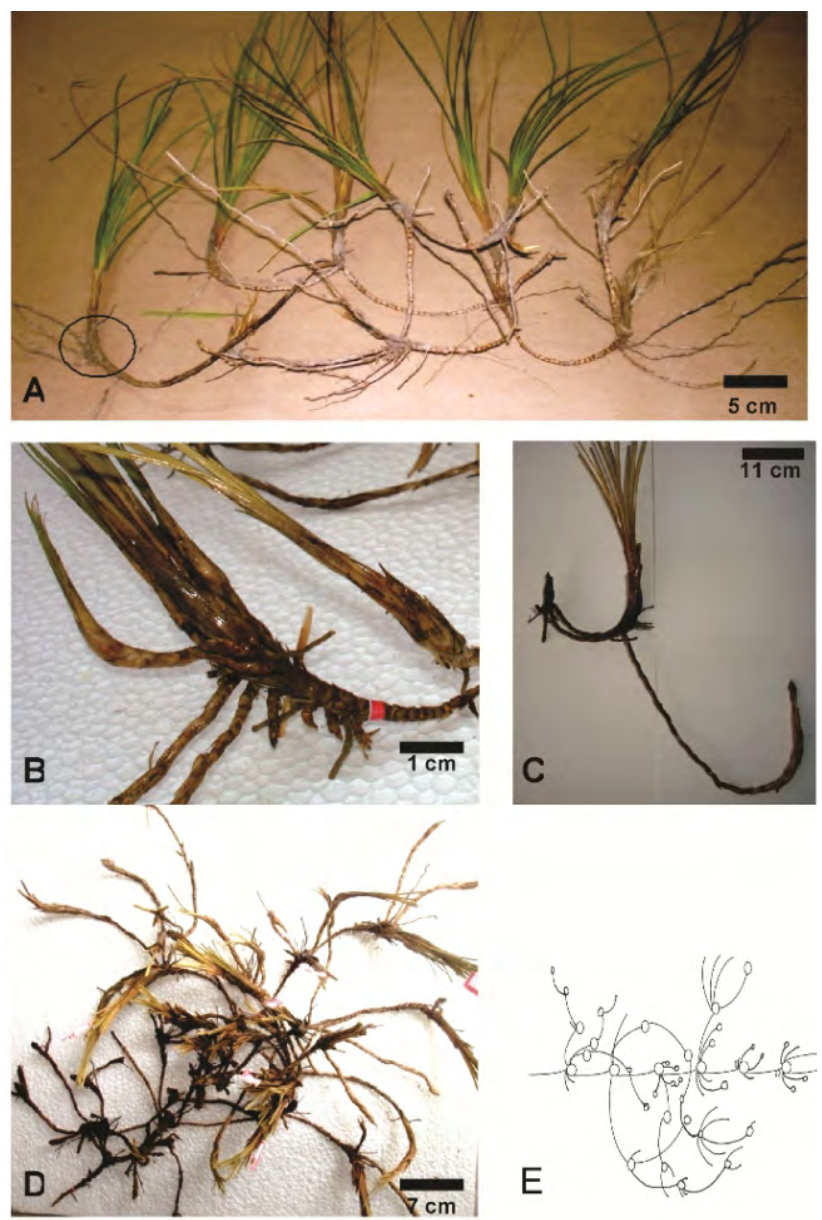

Figura 3. Rizomas de Hypolytrum pulchrum coletados no Orquidário do Pai Inácio, município de Palmeiras, Bahia, Brasil: A- arcos regulares e raízes saindo perpendicularmente da região nodal na base do ramo; Bdetalhe do arco com caules laterais, formado por meristemas laterais, nós e entrenós protegidos por catafilos; C- arco de $28 \mathrm{~cm}$. D-E- sistema de arcos em expansão e diagrama de crescimento.

Segundo Bell \& Tomlinson (1980), a propagação vegetativa, na qual módulos novos são estabelecidos a distâncias variadas do eixo parental através do comprimento de suas ramificações no substrato, é mais eficiente e econômica. A presença de muitas gemas nos arcos permite uma emissão simultânea de novos arcos. As folhas periféricas dos módulos morrem com o tempo e o resto das bainhas sobrepostas forma um revestimento fibroso que pode permanecer por anos na planta, conferindo proteção contra o fogo às gemas e mantendo a umidade central, uma parte soterrada e uma exposta, até que uma nova queimada elimine o excesso (Rachid-Edwards 1956). A biomassa aérea dessa espécie apresentou para uma unidade modular 5,425 g e biomassa subterrânea $0,902 \mathrm{~g}$, incluindo caule e raiz. Estes valores podem variar de acordo com o estádio de desenvolvimento dos módulos, dentre outros fatores.

Eryngium paraguariense. Apresenta um rizoma de consistência lenhosa, com formação contínua das folhas e ramificação simpodial resultante do tipo de propagação vegetativa (Figura 4). O sistema subterrâneo é espessado e curto, o que pode ser explicado pelo direcionamento de recursos para parte aérea, resultando em um índice $\alpha^{2}>1$, que representa elevado investimento aéreo para razão massa seca aérea/ massa seca subterrânea (Tabela 1). O encurtamento de entrenós pode ser resultante também da diminuição do metabolismo devido a condições climáticas (Bell \& Tomlinson 1980).

O sistema subterrâneo de E. paraguariense apresenta raízes adventícias intracorticais que se espalham radialmente ao longo do eixo, atingindo raio médio de 24,6 $\mathrm{cm}$ de comprimento (Figura 4; Tabela 1). Nessa espécie, o crescimento agregado dos módulos permite a formação de grandes rosetas que servem de proteção contra o fogo e acúmulo de água. É também funcional para espécies herbáceas de menor porte que crescem a partir da abertura gerada na matriz da vegetação (Fidelis et al. 2008, 2009). Essa arquitetura integrada aéreo-subterrâneo na qual a forma em roseta aproxima as raízes com a base das folhas, onde as gemas ficam protegidas, parece maximizar a velocidade no aproveitamento de pulsos de nutrientes e de disponibilidade de espaço.

$O$ padrão de crescimento agregado de $E$. paraguariense provavelmente tem relação com a propagação vegetativa observada, caracterizada pelo crescimento de módulos radiais ao rizoma, sendo que muitas vezes os sistemas curtos e espessados permanecem ligados, parecendo impedir a
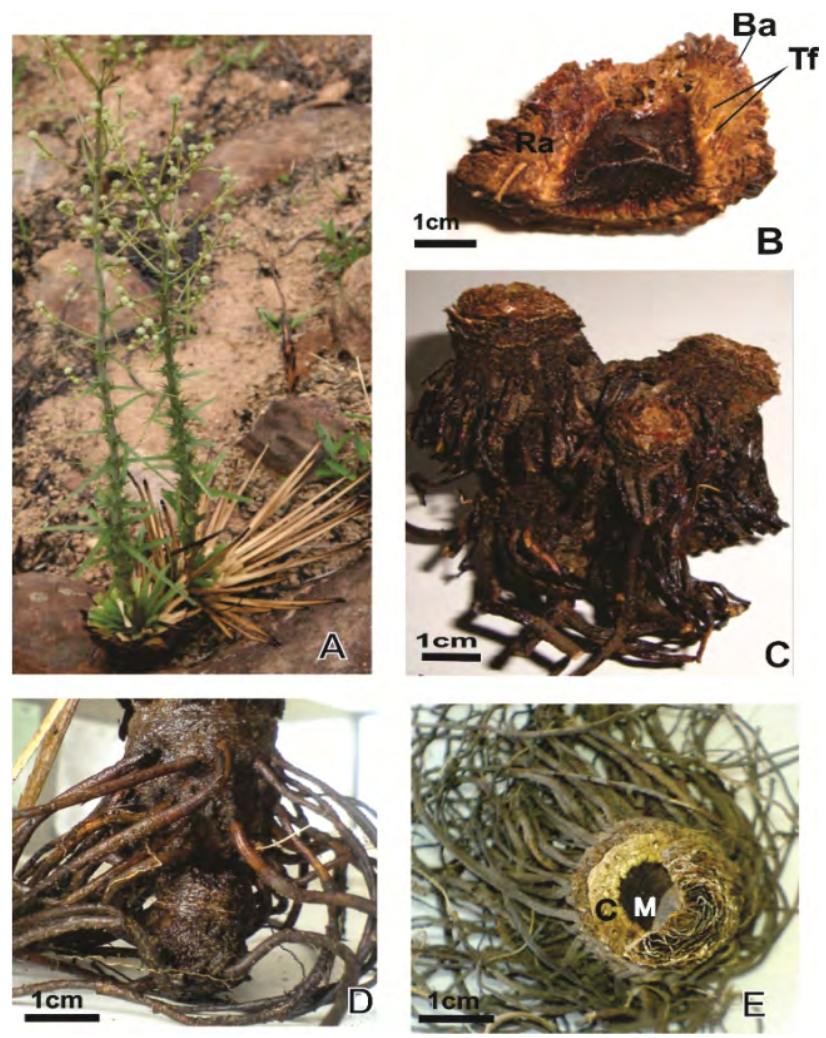

Figura 4. Amostras de Eryngium paraguariense coletadas no Orquidário do Pai Inácio, município de Palmeiras, Bahia, Brasil: A- planta no campo rupestre, cercado por rochas afloradas, hábito monocotiledôneo; B- parte superior do sistema subterrâneo em corte longitudinal radial (Tf- traço foliar; Ba- bainha foliar; Ra- raiz adventícia intracortical); C- rizomas interligados, formando padrão agregado com ramificação simpodial; Ddistribuição radial das raízes; $\mathbf{E}$ - rizoma visto de cima, em corte transversal com medula (M) e córtex visíveis (C), módulo monopolar. 


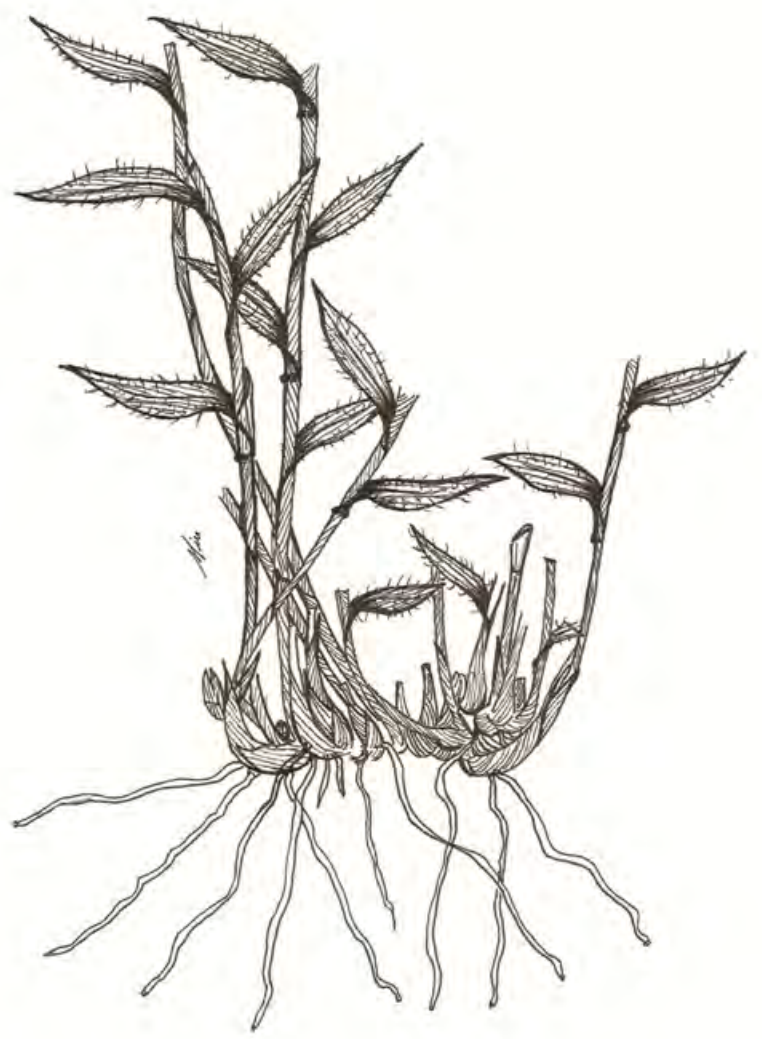

Figura 5. Ramos aéreos com bainha e rizoma revestido por bainha e raízes homorrízicas, de amostra de Echinolaena inflexa coletada no Orquidário do Pai Inácio, município de Palmeiras, Bahia, Brasil.

propagação de muitas gemas de uma só vez, o que não ocorre com as gramíneas onde a emissão de gemas é intensa, simultânea e aparentemente desorganizada. Essas gemas podem ter uma função importante no desenvolvimento de novos ramos após a floração e subsequente senescência da roseta. Essa possibilidade já foi apontada para outra espécie rizomatosa do mesmo gênero, Eryngium horridum Malme (Fidelis et al. 2008), pois devido à formação e morte do escapo, novos módulos são produzidos, mantendo a funcionalidade do sistema. Essa forma de crescimento está vinculada à fenologia da espécie, sendo também funcional diante de fenômenos externos que causem danos ao meristema apical, como herbivoria ou fogo (Coutinho 1977, 1982). Isto corrobora a ideia de que o padrão de propagação vegetativa e a população de gemas nos sistemas subterrâneos em fisionomias abertas guiam a dinâmica da comunidade vegetal sobre o solo (Appezzatoda-Glória \& Cury 2011).

Euphorbia sarcodes. Apresenta alocação preferencial de recursos para o sistema subterrâneo radicular, inverso ao caso de E. paraguariense (Figura 6; Tabela 2). Essa distribuição de recursos pode estar relacionada a vários fatores, como sazonalidade, forma de vida, fenologia,
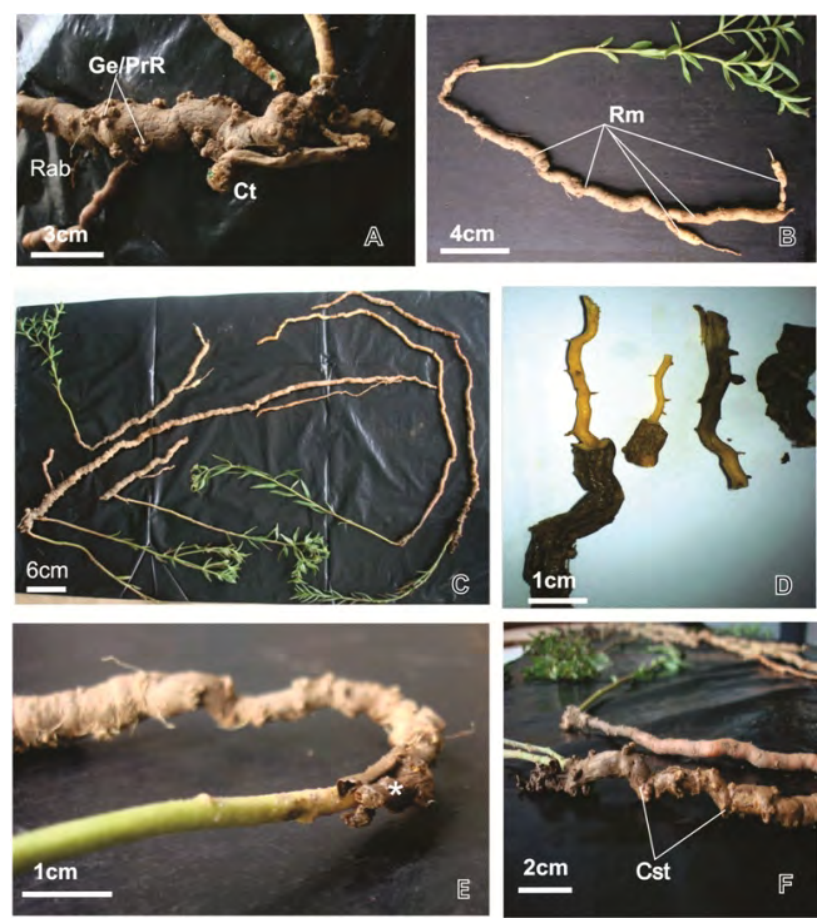

Figura 6. Amostras de Euphorbia sarcodes coletadas no Orquidário do Pai Inácio, município de Palmeiras, Bahia, Brasil. A- detalhe das gemas (Ge) ou primórdios radiculares (PrR) rodeadas por raízes de absorção (Rab) e cicatriz de rebroto (Ct); B- sistema com ramificações (Rm); Ccinco amostras de $E$. sarcodes; D- ramificações no cilindro vascular de diferentes partes da raiz, onde se posicionam gemas endógenas ou primórdios radiculares; E- cicatriz deixada pelo ramo velho em detalhe $(*) ; \mathbf{F}$-raiz e suas contrações (Cst)

Tabela 1. Parâmetros morfológicos e biomassa de Eryngium paraguariense. MAS- massa seca aérea, MSS- massa seca subterrânea, DESVPAD- desvio padrão.

\begin{tabular}{lllllllll}
\hline & $\begin{array}{l}\text { Eixo } \\
\text { caulinar } \\
(\mathrm{cm})\end{array}$ & $\begin{array}{l}\text { Raio das } \\
\text { raízes } \\
(\mathrm{cm})\end{array}$ & $\begin{array}{l}\text { Comprimento } \\
\text { das folhas } \\
(\mathrm{cm})\end{array}$ & $\begin{array}{l}\mathrm{MSA}(\mathrm{g}) \\
\text { Amostra }\end{array}$ & $\mathrm{MSS}(\mathrm{g})$ & $\begin{array}{l}\mathrm{MSA} / \mathrm{MSS} \\
\left(\alpha^{2}\right)(\mathrm{g})\end{array}$ & $\begin{array}{l}\text { Padrão de } \\
\text { crescimento no solo }\end{array}$ \\
\hline 1 & 5,5 & 20 & 85 & 134 & 34 & 3,94 & Isolado & Vertical \\
2 & 5 & 25 & 95 & 172 & 28 & 6,14 & Agregado & Vertical \\
3 & 4 & 27 & 90 & 142 & 26 & 5,46 & Agregado & Vertical \\
4 & 5,5 & 26 & 96 & 284 & 26 & 10,9 & Agregado & Vertical \\
5 & 4,5 & 25 & 90 & 160 & 30 & 5,3 & Agregado & Vertical \\
Média & 4,9 & 24,6 & 91,2 & 178,4 & 28,8 & 6,34 & - & - \\
DESVPAD & 0,65 & 2,7 & 4,44 & 60,9 & 3,34 & 2,66 & - & - \\
\hline
\end{tabular}


Tabela 2. Padrão morfológico e biomassa seca de Euphorbia sarcodes. CS- comprimento subterrâneo, CA- comprimento aéreo, MAS- massa seca aérea, MSS- massa seca subterrânea, MSF- massa seca foliar, MSC- massa seca caulinar, **** presença de inflorescência, *** raiz incompleta, ** dois ramos.

\begin{tabular}{|c|c|c|c|c|c|c|c|c|c|}
\hline Amostras & $\begin{array}{l}\mathrm{N}^{\circ} \text { de } \\
\text { ramificações }\end{array}$ & $\begin{array}{l}\mathrm{CS} \\
(\mathrm{cm})\end{array}$ & $\begin{array}{l}\mathrm{CA} \\
(\mathrm{cm}) \\
\end{array}$ & $\begin{array}{l}\mathrm{CA} / \mathrm{CS} \\
(\mathrm{cm})\end{array}$ & MSS $(g)$ & $\operatorname{MSF}(g)$ & $\operatorname{MSC}(\mathrm{g})$ & $\begin{array}{l}\text { MSA/MSS } \\
(\mathrm{g}) \alpha^{1}\end{array}$ & MSS/CS(g) \\
\hline 1 & 1 & 105 & 38 & 0,36 & 17,2 & $0,78^{* *}$ & $1,97^{* *}$ & 0,16 & 0,16 \\
\hline $2^{\star \star \star \star}$ & - & $66^{\star * *}$ & 38 & 0,57 & $5,83^{\star \star *}$ & 0,39 & 0,49 & 0,15 & 0,08 \\
\hline 3 & 5 & 33 & 25 & 0,75 & 6,15 & 0,28 & 0,49 & 0,12 & 0,18 \\
\hline 4 & - & 58 & 30 & 0,51 & 8,56 & 0,42 & 0,05 & 0,1 & 0,14 \\
\hline $5^{* * * *}$ & 1 & $17^{* * *}$ & 29 & 1,7 & $3,15^{\star \star *}$ & 0,66 & 0,87 & 0,48 & 0,18 \\
\hline Média & & 55,8 & 32 & 0,77 & 8,18 & 0,50 & 0,774 & 0,20 & 0,148 \\
\hline DESVPAD & & 33,7 & 5,7 & 0,53 & 5,4 & 0,20 & 0,72 & 0,15 & 0,04 \\
\hline
\end{tabular}

nutrição, competição, relações hídricas, hábito de crescimento, etc. (Brouwer 1962; Briske 1986; Raventos \& Silva 1988; Sarmiento 1992), aliados a heterogeneidade ambiental e eventos como fogo e secas (Conceição 2006; Neves \& Conceição 2010).

Um menor quociente da relação biomassa aérea/subterrânea pode significar um sistema radicular mais extenso e, possivelmente, mais eficiente (Buttery \& Bizzell 1972 apud Scheffer-Basso et al. 2002), como o apresentado em E. sarcodes (Tabela 2). No entanto, o aumento do comprimento da raiz não representa necessariamente aumento da capacidade de absorção de água e nutrientes (Kramer 1983). A eficiência em absorver água e nutrientes está vinculada à produção constante de raízes com alta capacidade de absorção (Horn et al. 2006). Espécies com um investimento inicial relativamente pequeno em biomassa por comprimento de unidade de raiz podem ter como vantagem explorar pulsos de água ou nutrientes no solo, principalmente em ambientes perturbados (Eissenstat 1991). Euphorbia sarcodes possui uma elevada biomassa de raiz $\left(\alpha^{1}\right)$, embora a biomassa por comprimento de raiz seja baixa (Tabela 2). Apresenta muitos primórdios radiculares ao longo da área de superfície da raiz que podem estar relacionados com a sazonalidade, uma vez que a coleta foi realizada no início da estação seca. Na estação chuvosa, os primórdios devem desenvolver-se e formar as raízes laterais que irão explorar o solo em extensão. Logo, o desprendimento de energia para explorar a superfície em extensão pode estar sendo utilizado na época seca para exploração em profundidade. O fato da raiz não explorar a extensão da superfície onde fica a maior parte dos nutrientes não representa necessariamente uma condição negativa para a planta, pois ela pode apresentar maior eficiência no aproveitamento de nutrientes em solos pobres.

Euphorbia sarcodes apresenta órgão subterrâneo gemífero contendo látex, raiz pivotante com crescimento vertical e pequenas contrações na superfície (Figura 6).
Essas características foram observadas em áreas de solo com baixa resistência de rochas e alta presença de cupins (até $50 \mathrm{~cm}$ de profundidade), que favorecem a aeração do solo e facilita o crescimento vertical da raiz. Dessa forma, a planta garante acesso à água mais profunda, driblando a competição e favorecendo sua sobrevivência em períodos mais secos. Além disso, os cupins proporcionam condições nutricionais diferenciadas no local (Holt \& Lepage 2000). Essa distribuição espacial de organismos no solo pode ter efeito direto e indireto, influenciando na dinâmica e estrutura da comunidade vegetal (Ettema \& Wardle 2002). Segundo Appezzato-da-Glória (2003), a contração radicular permite a ocorrência de gemas em solos mais profundos, um mecanismo favorável em condições ambientais adversas. A propagação vegetativa nessa espécie pode estar relacionada à capacidade de modificação da arquitetura frente a perturbações. Dessa maneira, $E$. sarcodes apresenta um padrão morfoanatômico relacionado à sobrevivência em ambientes com condições edáfo-climáticos estressantes.

\section{CONSIDERAÇÕES FINAIS}

A heterogeneidade ambiental, herbivoria e secas são fatores ecológicos que, associados ao distúrbio por fogo, contribuem para a complexidade na estrutura e dinâmica da vegetação nos campos rupestres. As plantas apresentam arranjos espaciais que permitem a elas coexistirem através de um jogo de habilidades associadas a características morfofuncionais de partes aérea e subterrânea integradas. A morfologia é um aspecto importante que ajuda a entender respostas competitivas. Nas espécies que apresentaram sistema subterrâneo integrado, há alocação preferencial de recursos para partes aéreas, mas outros estudos são necessários para o entendimento dos processos que influenciam esses padrões e as respostas das plantas na comunidade vegetal.

\section{REFERÊNCIAS}

Apezzato-da-Glória, B. 2003. Morfologia de Sistemas

Subterrâneos: histórico e evolução do conhecimento no

Brasil. Ed. Alexandre Sene Pinto, Ribeirão Preto.

Appezzato-da-Glória, B. \& Cury, G. 2011. Morpho-anatomical features of underground systems in six Asteraceae species from the Brazilian Cerrado. Annals of the Brazilian Academy of Sciences 83(3): 981-991.

Bell, A.D. \& Tomlinson, P.B. 1980. Adaptive architecture in 
rhizomatous plants. Botanical Journal of Linnean Society 80: 125-160.

Briske, D.D. 1986. Plant response to defoliation: morphological consideration and allocation priorities. In: P.J. Joss, P.W. Lynch \& O.B. Williams (eds), Rangelands: a resource under siege. Cambridge University Press, Cambridge, p. 425-427.

Brouwer, R. 1962. Distribution of dry matter in the plant. Netherlands Journal of Agricultural Science 10: 361-376.

Cahill Jr, J.F. 2003. Lack of relationship between below-ground competition and allocation to roots in 10 grassland species. Journal of Applied Ecology 91(4): 532-540.

Casper, B.B. \& Jackson, R.B. 1997. Plant Competition Underground. Annual Review of Ecology, Evolution, and Systematics 28: 545-570.

Cianciaruso, M.V.; Silva, I.A \& Batalha, M.A. 2010. Aboveground biomass of functional groups in the ground layer of savannas under different fire frequencies. Australian Journal of Botany 58(3): 169-174.

Conceição, A.A. 2006. Plant ecology in 'campos rupestres' of the Chapada Diamantina, Bahia. In: L.P. Queiroz, A. Rapini \& A.M. Giulietti (eds), Towards Greater Knowledge of the Brazilian Semi-arid Biodiversity. Ministério da Ciência e Tecnologia, Brasília, p. 63-67.

Conceição, A.A. \& Giulietti, A.M. 2002. Composição florística e aspectos estruturais de campo rupestre em dois platôs do Morro do Pai Inácio, Chapada Diamantina, Bahia, Brasil. Hoehnea 29(1): 37-48.

Costa, G.M. 2010. Regeneração da Vegetação de Campo Rupestre sob a Perturbação de Fogo na Chapada Diamantina, Bahia. Dissertação (Mestrado em Botânica). Universidade Estadual de Feira de Santana.

Coutinho, L.M. 1977. Aspectos ecológicos do fogo no cerrado. II. As queimadas e a dispersão de sementes em algumas espécies anemocóricas do estrato herbáceo-subarbustivo. Boletim de Botânica da Universidade de São Paulo 5: 57-64.

Coutinho, L.M. 1982. Ecological effects of fire in the Brazilian Cerrado. In: B.J. Huntley \& B.H. Walker (eds), Ecology of Tropical Savannas. Springer-Verlag, Berlin, p. 81-105.

Eissenstat, D.M. 1991. On the relationship between specific root length and rate of root proliferation: a field study using citrus rootstocks. The New Phytologist 69: 870-873.

Ettema, C.H. \& Wardle, D. 2002. Spatial soil ecology. Trends in Ecology and Evolution 17: 177-183.

Fidelis, A.; Overbeck, G.; Pillar, V.D. \& Pfadenhauer, J. 2008. Effects of disturbance on population biology of the rosette species Eryngium horridum Malme in grasslands in southern Brazil. Plant Ecology 195: 55-67.

Fidelis, A.; Overbeck, G.; Pillar, V.D. \& Pfadenhauer, J. 2009. The ecological value of Eryngium horridum in maintaining biodiversity in subtropical grasslands. Austral Ecology 34: 558-566.

Fitter, A.H. \& Stickland, T.R. 1991. Architectural analysis of plant root systems. 3. Studies on plants under field conditions. New Phytologist 121:243-248.

Grams, T.E.E. \& Andersen, C.P. 2007. Competition for resources in trees: physiological versus morphological plasticity. Progress in Botany 68: 356-381.

Grillo, A.A.S. 2008. Cerrado: áreas do Cercado e do Morro do Camelo. In: L.S. Funch, R.R. Funch \& L.P. Queiroz (eds), Serra do Sincorá: Parque Nacional da Chapada Diamantina. Radami, Feira de Santana, p. 79-101.

Holt, J.A. \& Lepage, M. 2000. Termite and soil properties. In: T. Abe, D.E. Bignell \& M. Hihashi (eds), Termites: evolution, sociality, symbiosis, ecology. Kluwer Academic Publishers,
Dordrecht, p 389-407.

Horn, D.; Ernani, P.R.; Sangoi, L.; Schweitzer, C \& Cassol, P.C. 2006. Parâmetros cinéticos e morfológicos da absorção de nutrientes em cultivares de milho com variabilidade genética contrastante. Revista Brasileira de Ciência do Solo 30(1): 77-85.

Klink, C.A. 1994. Effects of clipping of size and tillering of native and African grasses of the Brazilian savannas (the Cerrado). Oikos 70: 365-376.

Klink, C.A. \& Silva, D.A. 2001. Dinâmica de foliação e perfilhamento de duas gramíneas $\mathrm{C} 4$ e uma $\mathrm{C} 3$ nativas do Cerrado. Revista Brasileira de Botânica 24(4): 441-446.

Kolbek, J. \& Alves, R.J.V. 2008. Impacts of cattle, fire and wind in rocky savannas, southeastern Brazil. Acta Universitatis Carolinae Environmentalica 22: 111-130.

Kramer, P.J. 1983. Development of root system. Water Relations of Plants. Academic Press, New York, p. 146-186.

Menezes, N.L.; Muller, C. \& Sajo, M.G. 1979. Um novo e peculiar tipo de sistema subterrâneo em espécies de Vernonia da Serra do Cipó (Minas Gerais, Brasil). Boletim de Botânica da Universidade de São Paulo 7:33-38.

Negreiros, D.; Fernandes, G.W.; Silveira, F.A.O. \& Chalub, C. 2009. Seedling growth and biomass allocation of endemic and threatened shrubs of rupestrian fields. Acta oecologica 35: $301-310$

Neves, S.P.S. \& Conceição, A.A. 2010. Campo rupestre recémqueimado na Chapada Diamantina, Bahia, Brasil: plantas de rebrota e sementes, com espécies endêmicas na rocha. Acta Botanica Brasilica 24(3): 697-707.

Nimer, N. 1989. Climatologia do Brasil. 2 ed. IBGE, Rio de Janeiro.

Oborny, B.; Kun, A.; Czárán, T \& Bokros, S. 2000. The effect of clonal integration on plant competition for mosaic habitat space. Ecology 81(12): 3291-3304.

Rachid-Edwards, M. 1956. Alguns dispositivos para proteção de plantas contra a seca e o fogo. Boletim de Botânica da Universidade de São Paulo 13: 37-69.

Raventos, J. \& Silva, J.F. 1988. Architecture, seasonal growth and interference in three grass species with different flowering phenologies in a tropical savanna. Vegetation 75 : 115-123.

Sarmiento, G. 1992. Adaptive strategies of perennial grasses in South American savannas. Journal of Vegetation Science 3: 325-336.

Scheffer-Basso, S.M.; Agnol, M.D. \& Jacques, A.V.A. 2002. Alocação da biomassa e correlações morfofisiológicas em leguminosas forrageiras com hábitos de crescimento contrastantes. Scientia Agricola 59(4): 629-634.

Silveira, M. 2001. A Floresta Aberta com Bambu no Sudoeste da Amazônia: padrões e processos em múltiplas escalas. Tese (Doutorado em Ecologia), Universidade de Brasília.

Souza, A.; Sandrin, C.Z.; Calió, M.F.A.; Meirelles, S.T.; Pivello, V.R. \& Figueiredo-Ribeiro, R.C.L. 2010. Seasonal variation of soluble carbohydrates and starch in Echinolaena inflexa, a native grass species from the Brazilian savanna, and in the invasive grass Melinis minutiflora. Brazilian Journal of Biology 70(2): 395-404.

Tilman, D. 1982. Resource Competition and Community Structure. Monographs (Population Biology). Princeton University Press, Princeton.

Van Groenendael, J. M.; Klimes, L.; Klimesová, J. \& Hendriks, R.J.J. 1996. Comparative ecology of clonal plants. Philosophical Transactions of the Royal Society of London B 351: 1331-1339. 